\title{
COVID-19 pandemic alert: time to focus on lung health-Beijing call to action for lung health promotion
}

\author{
Global Alliance of Chronic Respiratory Diseases (GARD) \\ Correspondence to: Arzu Yorgancioglu. GARD Chair, Department of Pulmonology, Medical Faculty, Celal Bayar University, Manisa, Turkey. \\ Email: arzuyo@hotmail.com.
}

Submitted May 07, 2020. Accepted for publication Jun 09, 2020.

doi: 10.21037/jtd-gard-20-002

View this article at: http://dx.doi.org/10.21037/jtd-gard-20-002

Since December, 2019, the COVID-19 has influenced global health with more than 7 million confirmed cases and more than 400,000 death (1). This contagious virus spreads from person to person through aerosol inhalation and contact, indicating that like many other infectious disease, such as influenza or SARS or MERS, it is urgent and critical to focus on lung health and promoting people stay heathy.

Global headlines on lung health are often limited to Tuberculosis. Unlike heart or liver lungs do not get their due in the global and national health agendas. More than $80 \%$ of the people living in urban areas that monitor air pollution are exposed to air quality that exceed WHO guideline limits. Chronic respiratory diseases (CRD) chronic obstructive pulmonary disease (COPD) and Asthma-are a major cause of death worldwide, significantly contributing to the global burden from noncommunicable diseases (NCDs). In 2017, an estimated 3.2 million deaths were caused by COPD (2). Tobacco smoking, indoor and outdoor air pollution, and a rapidly aging population are likely to increase the burden of CRD and more efforts are needed for prevention and with health promotion across the life course. In addition, for COVID-19 pandemic, around 5\% COPD/asthma patients coinfected with SARS$\mathrm{CoV}-2$ with high mortality and morbidity (3). Smoking increases ACE2 expression and make the COPD patients more vulnerable to SARS-CoV-2 infection (4) together with downregulated immune response to viral infection of COPD patients. Thus, for COPD patients during the current pandemic, stay home, avoid public exposure, wash hands and keep room ventilation, as well as keeping sufficient COPD treatment medicine available is crucial strategy to maintain their heathy status.

National guidelines are not sufficiently defined, disseminated and adhered to, leading to suboptimal outcomes of treatment. Management at the primary healthcare level is challenged by the lack of medicines and personnel.

Global Alliance against Respiratory Diseases (GARD) is a voluntary alliance of national and international organizations, institutions, and agencies committed towards the common goal to reduce the global burden of respiratory diseases. The $13^{\text {th }}$ general meeting of GARD was held in Beijing from the $26^{\text {th }}$ to $27^{\text {th }}$ of October 2019. The meeting adopted the 'Beijing Call to Action for Lung Health Promotion' with 4 key priority areas.

\section{Advocating for action on chronic respiratory diseases}

* Advocate at all levels of government to scale actions towards the achievement of the Sustainable Development Goal 3.4, ensuring that CRDs are firmly placed in the national response to NCDs.

* Ensure that leaders at all levels take responsibility for comprehensive local actions, together with the health sector, that can advance action for the control of CRD, as well as other NCDs.

- Acknowledge and support vulnerable high-risk populations, disproportionally exposed to modifiable risk factors, as a result of their gender, age, disability, socioeconomic status, education, sociocultural context, and any other factor that may influence their exposure to risk factors and their access to health services.

\section{Fostering multisectoral action to reduce risk factors for CRDS}

* Engage with multiple stakeholders to prioritize sustainable and long-term actions against CRD, through 


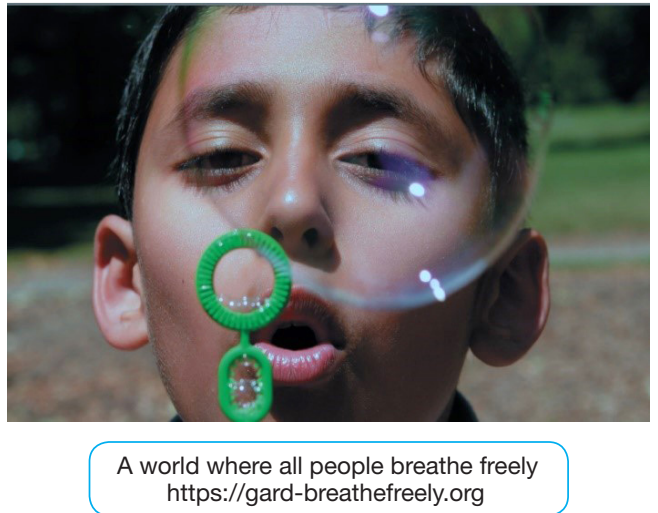

'whole-of-government' and 'whole-of-society' initiatives, and mainstreaming CRD prevention in all policies.

* Advocate for increasing excise taxes and prices on tobacco products, as well as for implementing other costeffective interventions, to reduce the high burden of tobacco and the alarmingly rapid spread of Electronic Nicotine Delivery Systems.

* Promote intersectoral action to address indoor and outdoor air pollution, by engaging with a range of other sectors and identifying clean-energy strategies to shift away from kerosene, biomass and biofuel burning, and to reduce $\mathrm{CO}_{2}$ emissions.

\section{Strengthening primary health care for CRD to achieve universal health coverage}

* Ensure that national Universal Health Coverage benefit packages include CRD services, including respiratory health promotion and prevention, as well as access to essential medicines and technologies, through the adaptation of the WHO Model Lists of Essential Medicines and Essential in vitro Diagnostics.

* Strengthen primary health services on CRD to ensure equitable coverage, including essential public health functions, such as tobacco cessation programs, pneumococcal and influenza vaccinations, with an adequate and well-equipped multi-disciplinary health workforce, based on task-shifting and task-sharing approaches.

* Identify opportunities in chronic-care platforms and promote an integrated and holistic approach to CRD, taking into account that patients often suffer from several conditions, simulating and/or exacerbating lung diseases, such as tobacco dependence, allergy, pneumonia, tuberculosis, cardiovascular diseases, obesity, upper airway conditions, cancers, severe mental illness, depression and anxiety.

* Prioritize respiratory health and CRD prevention and control in the formal training provided to health professionals.

* Adapt standardized interventions for the management of CRDs at the PHC level, drawing from evidence-based recommendations and tools, such as the WHO Package of Essential NCD interventions.

* Empower patients with CRD in advocacy and self-care, including using appropriate tools, such as mobile health (mHealth).

* Recognize the importance of referral care and secure fast track for CRD patients who need respiratory specialist diagnoses and treatments, including pulmonary rehabilitation.

\section{Supporting research}

* Invest in identifying bottlenecks and challenges to the large-scale implementation of interventions to prevent $\mathrm{CRD}$, in order to determine feasible solutions.

* Invest in translational and health system research for global application of proven cost-effective strategies especially in resource-constrained settings.

* Develop and implement internationally recognized indicators, at the health facility, sub-national, and national levels, to measure the burden of CRD and to monitor the quality of provided services and progress in averting CRD premature deaths.

* Investigate the intersection between social and environmental determinants of health and their impact on the management of CRD, with emphasis on the role of populations in conflict.

\section{Acknowledgments}

We thank GARD members and GARD delegate (https:// www.who.int/gard/en/) to contribute to this document and GARD staff who prepared this manuscript, including Wang Chen (Peking Union Medical College, Beijing, China), Cherian Varghese (GARD Secretariat, Management of NCDs, WHO, Geneva, Switzerland), Arzu Yorgancioglu (GARD Chair, Department of Pulmonology, Medical Faculty, Celal Bayar University, Manisa, Turkey), Giuseppe Troisi (GARD Secretariat, Management of NCDs, WHO, Geneva, Switzerland), Mina Gaga (Respiratory Medicine Department, Asthma Cen, Athens Chest Hospital, Athens), Talant Sooranbaev (Kyrgyzstan National Centre of 
Cardiology and Internal Medicine, Euro-Asian Respiratory Society, Bishkek, Kyrgyzstan), Teresa To (The Hospital for Sick Children, Dalla Lana School of Public Health, University of Toronto, Ontario, Canada); WHO GARD Planning Group [To Teresa (The Hospital for Sick Children, Dalla Lana School of Public Health, University of Toronto, Ontario, Canada), Viegi Giovanni (CNR Institutes of Clinical Physiology, Pisa, and Biomedical Research and Innovation, Palermo, Italy), Cruz Alvaro (ProAR Foundation and Federal University of Bahia, Salvado, Brazil), Asher Innes (Department of Paediatrics and Child Health, University of Auckland, New Zealand), Behera Digamber (OSD, All India Institutes of Medical Sciences (AIIMS), Raebareli, India), Bennour Kazi (Bangladesh Lung Foundation, Bangladesh), Boulet Louis Philippe (Québec heart and Lung Institute, Laval University, Québec, Canada), Bousquet Jean (MACVIA-France, Fondation Partenariale FMC VIA-LR, CHRU Arnaud de Villeneuve, Montpellier, France), Camargos Paolo (Universidade Federal de Minas Gerais, Faculdade de Medicina, Departamento de Pediatria, Belo Horizonte, Minas Gerais, Brazil), Conceiçao Claudia (Instituto de Higiene e Medicina Tropical, Institute of Tropical Medicine and Hygiene, NOVA University of Lisbon, Portugal), Diaz Sandra Gonzales (Hospital Universitario y Facultad de Medicina, Monterrey Nuevo Leon, Mexico), El Sony Asma (Public Health Epidemiological Laboratory [Epi Lab] for Research and Development, Khartoum, Sudan), Erhola Marina (Division of Health and Social Service, Finnish Institute for Health and Welfare, Helsinki, Finland), Gaga Mina (Respiratory Medicine Department, Asthma Cen, Athns Chest Hospital, Athens), Halpin David (University of Exter Medical School, College of Medicine and Health, Exeter, UK), Harding Letitia (Asthma and Respiratory Foundation, New Zealand), Maghlakelidze Tamaz (Ivane Javakhishvili Tbilisi State University, Pulmonology Department, Chapidze Emergency Cardiology Center, Tbilisi, Georgia), Masjedi Muhammed Reza (Shahid Beheshti University of Medical Sciences, Tehran, Iran), Mohammad Yousser (National Center for Research inn Chronic Respiratory Diseases, Tishreen University, School of Medicine, Latakia, Syria), Nunes Elizabete (Pulmonology Department, Maputo Central Hospital, Maputo, Mozambique), Pigearias Bernard (Espace Francophone de Pneumologie, Paris, France), Sooronbaev Talant (Kyrgyzstan National Centre of Cardiology and Internal Medicine, Euro-Asian Respiratory Society, Bishkek, Kyrgyzstan), Stelmach Rafael
(ProAR Foundation and Heart Institute (InCor) Hospital das Clinicas, Faculdade de Medicina, Universidade de Sao Paulo, Brazil), Taborda-Barata Luis (Department of Allergy \& Clinical Immunology, Cova da Beira University Hospital Centre, Covilhã, Portugal), Tuyet Lan Le Thin (Respiratory Care Center, University Medical Center, Ho Chi Minh City, Vietnam), Valiuis Arunas (Clinic of Children's Diseases, Institute of Clinical Medicine, and Department of Public Health, Institute of Health Sciences, Vilnius University, Vilnius, Lithuania), Wang Chen (Peking Union Medical College, Beijing, China), Williams Sian (International Primary Care Respiratory Group, London, UK), Yorgancioglu Arzu (Department of Pulmonology, Medical Faculty, Celal Bayar University, Manisa, Turkey), Cherian Varghese (GARD Secretariat, Management of NCDs, WHO, Geneva, Switzerland)].

Funding: None.

\section{Footnote}

Provenance and Peer Review: This article was commissioned by Guest Editors (Yousser Mohammad and Alvaro A. Cruz) and the editorial office of Fournal of Thoracic Disease for the series "GARD Section" published in Journal of Thoracic Disease. The article did not undergo external peer review.

Conflicts of Interest: All authors have completed the ICMJE uniform disclosure form (available at http://dx.doi. org/10.21037/jtd-gard-20-002). The series "GARD Section" was commissioned by the editorial office without any funding or sponsorship. The authors have no other conflicts of interest to declare.

Ethical Statement: The authors are accountable for all aspects of the work in ensuring that questions related to the accuracy or integrity of any part of the work are appropriately investigated and resolved.

Open Access Statement: This is an Open Access article distributed in accordance with the Creative Commons Attribution-NonCommercial-NoDerivs 4.0 International License (CC BY-NC-ND 4.0), which permits the noncommercial replication and distribution of the article with the strict proviso that no changes or edits are made and the original work is properly cited (including links to both the formal publication through the relevant DOI and the license). See: https://creativecommons.org/licenses/by-nc-nd/4.0/. 


\section{References}

1. Available online: https://www.who.int/emergencies/ diseases/novel-coronavirus-2019/situation-reports

2. Available online: https://indicators.report/targets/3-4/

3. Guan WJ, Ni ZY, Hu Y, et al. Clinical Characteristics of Coronavirus Disease 2019 in China. N Engl J Med 2020;382:1708-20.

4. Leung JM, Yang CX, Tam A, et al. ACE-2 expression in the small airway epithelia of smokers and COPD patients: implications for COVID-19. Eur Respir J 2020;55:2000688.
Cite this article as: Global Alliance of Chronic Respiratory Diseases (GARD). COVID-19 pandemic alert: time to focus on lung health-Beijing call to action for lung health promotion. J Thorac Dis 2020;12(6):3238-3241. doi: 10.21037/jtdgard-20-002 